\title{
Adjuvant chemotherapy with 5-FU or regimens including oral fluoropyrimidine for curable gastric cancer
}

\author{
Mitsuru SAsako \\ Department of Surgery, Hyogo College of Medicine, 1-1-Mukogawa-cho, Nishinomiya, Hyogo 663-8501, Japan
}

\begin{abstract}
Since 2000, several studies have reported positive results in reasonable-size randomized controlled trials of adjuvant treatment for potentially curable gastric cancer. At present, postoperative adjuvant chemoradiotherapy and perioperative chemotherapy are the standard of care in the United States and Europe (including Great Britain), respectively, while postoperative S-1 monotherapy is the standard of care in Japan. The effect of adjuvant treatment varies according to the type of surgery, and the best results so far have been observed in the adjuvant chemotherapy of TS-1 for gastric cancer (ACTS-GC) trial, in which D2 surgery followed by S-1 monotherapy was tested. The role of radiotherapy after D2 dissection remains unclear.
\end{abstract}

Key words Adjuvant treatment · Fluorouracil · Chemoradiotherapy $\cdot$ Gastric cancer

\section{Introduction}

A meta-analysis of the randomized controlled trials (RCTs) on adjuvant chemotherapy for curable gastric cancer reported the efficacy of the treatment in 2000 [1], although there had been no pivotal study showing the benefit of adjuvant treatment before 2000. In this century, however, several reports have presented the efficacy of adjuvant treatment for gastric cancer.

\section{Results of Western trials}

Intergroup study of adjuvant chemoradiotherapy [2]

Macdonald et al. [2] reported the results of the Intergroup 0116/South West Oncology Group (SWOG) 9008 study in 2001; the study was performed to evaluate the efficacy of adjuvant treatment comprising the adminis-

Offprint requests to: $\mathrm{M}$. Sasako

Received: March 24, 2008 / Accepted: April 24, 2008 tration of 45-Gy radiotherapy and five courses of chemotherapy consisting of 5-fluorouracil (5-FU) and leucovorin. Postoperative adjuvant chemoradiotherapy (CRT) showed a statistically significant improvement of relapse-free survival (RFS) and overall survival (OS) for patients with gastric cancer undergoing curative surgery, compared with surgery alone as control. The 3 -year OS after CRT was 50\%, while that of the surgeryalone group was $41 \%$ (hazard ratio [HR], 1.35; 95\% confidence interval [CI], 1.09-1.66; $P=0.005)$. The chemotherapy used in this study, 5-FU and leucovorin, was a slightly-out-of-date regimen, but the low toxicity and high compliance of this treatment could have been the key to this successful study.

The study had two major weak points. First, only $10 \%$ of patients underwent D2 dissection in spite of the recommendation of D2 dissection in the protocol, suggesting that poor local control by surgery was salvaged by radiotherapy. Secondly, $35 \%$ of the irradiation plans had major or minor deviations, most of which could be revised before actual treatment by the central quality controller. The eventual rate of major deviation was $6.5 \%$. This happened in the United States, where the standard level of radiotherapy seems to be much higher than that in most other countries, including Japan. This fact should be taken into consideration when this treatment is adopted in other countries.

After this result came out, the standard treatment after potentially curative surgery for node-positive patients in the United States has been postoperative CRT. At present, we cannot see any United States clinical phase III trial of adjuvant treatment for potentially curable gastric cancer in the registry of the NCI (http:// www.clinicaltrial.gov.)

\section{MAGIC trial [3]}

Cunningham et al. [3] reported the results of the MAGIC trial, which was performed to evaluate the efficacy of 
Table 1. List of reviewed trials

\begin{tabular}{|c|c|c|c|c|c|c|c|c|c|}
\hline \multirow[b]{2}{*}{ Author } & \multirow{2}{*}{$\begin{array}{l}\text { Accrual } \\
\text { period }\end{array}$} & \multirow{2}{*}{$\begin{array}{l}\text { No. of } \\
\text { patients } \\
\text { analyzed }\end{array}$} & \multirow[b]{2}{*}{ Chemotherapy } & \multicolumn{2}{|c|}{ 3-Year OS (\%) } & \multicolumn{2}{|c|}{ 5-Year OS (\%) } & \multirow[b]{2}{*}{ HR } & \multirow[b]{2}{*}{$P$ value } \\
\hline & & & & ACT & Surgery & $\mathrm{ACT}$ & Surgery & & \\
\hline Nakajima T [11] & 1988-1992 & 579 & $\mathrm{MMC}+5 \mathrm{FU}, \mathrm{UFT}$ & & & 85.8 & 82.9 & 0.738 & 0.17 \\
\hline Macdonald [2] & 1991-1998 & 556 & 5-FU+Leu, Rad (45 Gy) & 50 & 41 & & & 0.74 & 0.005 \\
\hline Nashimoto [12] & 1993-1994 & 252 & $\mathrm{MMC}+5 \mathrm{FU}+\mathrm{AraC}, \mathrm{UFT}$ & & & 91.2 & 86.1 & NA & 0.14 \\
\hline Neri [4] & 1989-1991 & 137 & Epirubicin+5FU+Leu & & & 30.2 & 12.6 & 0.51 & $<0.01$ \\
\hline Bajetta [6] & 1992-1997 & 271 & Etopo+Adria+CDDP & & & 52 & 48 & 0.93 & 0.87 \\
\hline Chipponi [7] & 1989-1997 & 199 & CDDP+5FU+Leu & & & 39.0 & 38.7 & NA & NA \\
\hline Bouche [8] & 1989-1997 & 260 & $5 \mathrm{FU}+\mathrm{CDDP}$ & & & 46.6 & 41.9 & 0.74 & 0.063 \\
\hline Nitti [9] & 1990-1998 & $191+206$ & FAMTX+Leu, FEMTX+Leu & & & 43 & 44 & 0.98 & 0.86 \\
\hline De Vita [10] & 1996-2001 & 225 & Epirubicin+Leu+5FU+Etopo & & & 48.0 & 43.5 & 0.91 & 0.61 \\
\hline Nakajima [14] & 1997-2001 & 190 & UFT & & & 86 & 73 & 0.48 & 0.017 \\
\hline Cunningham [3] & 1994-2002 & 503 & Epirubicin+CDDP+5FU & & & 36.3 & 23.0 & 0.75 & 0.009 \\
\hline Sakuramoto [15] & $2001-2003$ & 1059 & S-1 & 80.1 & 70.1 & & & 0.68 & 0.003 \\
\hline
\end{tabular}

ACT, adjuvant chemotherapy; HR, hazard ratio; MMC, mitomycin C; 5FU, 5-fluorouracil; Leu, leucovorin; Rad, radiation; AraC, cytarabine; Etopo, etoposide; Adria, adriamycin; CDDP, cisplatin; UFT, uracil-tegafur; FAMTX, 5-fluorouracil + adriamycin + methotrexate; FEMTX, 5fluorouracil + epirubicin + methotrexate; NA, not available

perioperative chemotherapy (three cycles each before and after surgery). The chemotherapy (ECF) used for this trial was a combination of epirubicin $\left(50 \mathrm{mg} / \mathrm{m}^{2}\right.$; day 1), cisplatin $\left(60 \mathrm{mg} / \mathrm{m}^{2}\right.$; day 1$)$, and 5 -FU [200 mg/ $\mathrm{m}^{2} /$ day; continuous intravenous administration (civ) days 1-21]. This treatment showed statistically significant improvement of both PFS and OS compared with surgery alone as control. The 5-year OS was $36.3 \%$ in the chemotherapy group and $23.0 \%$ in the surgeryalone group. There were 100 participating hospitals with no active quality control of surgery. Therefore, only about $53 \%$ of curable patients underwent D2 dissection. Secondly, $14.5 \%$ of the patients had adenocarcinoma of the esophagus, requiring a different type of surgery. Thirdly, shortly after randomization, 9 of 253 patients allocated to surgery alone did not undergo surgery or no information about surgery was available for them. If the quality of eligibility assessment had been reasonable, it would have been impossible that so many of the randomized patients did not undergo surgery. Fourthly, among 198 patients who underwent surgical resection, the pathological T stage was unknown in 5 patients and the pathological nodal stage was unknown in 42 patients. These facts strongly suggest that the quality of this trial was much poorer than those of the Intergroup 0116/SWOG 9008 study and Japanese studies. In the MAGIC trial, as the OS of curable patients in the surgery-alone group was not reported separately, comparison of results with those of other clinical trials which included only curable patients is almost impossible. However, the tumors resected in the control group were not more advanced than those included in the Intergroup 0116/SWOG 9008 study or in Japan Clinical Oncology Group (JCOG) studies.
Other clinical phase III trials with surgery alone as the control arm

In this century, six other articles reporting the results of RCTs of adjuvant chemotherapy with surgery alone as a control could be found. All but one included 5-FU as a component of the regimen. In 2001, Neri et al. [4] reported the results of a small RCT including 137 patients in total. The chemotherapy used was a combination of epirubicin, 5-FU, and leucovorin (EFL). In this paper, they reported a statistically significant survival benefit for chemotherapy over surgery alone $(P<0.01)$ [4]. However, the number reported in the interim analysis of the same trial published in 1996 [5] was different from the report of their final analysis [4], suggesting a low quality of this trial. None of the other five studies showed statistically significant differences between treatment and observation after surgery [6-10]. Table 1 shows the results of these trials. One of them was a combined analysis of two trials including 191 and 206 patients. The common aspect of these six trials is the limited number of patients enrolled in each study, fewer than 300 if combined analysis is divided by trial. None of these studies showed a statistically significant difference between the arms, which might have been reached if they had had 500 patients in one arm.

\section{Results of Japanese clinical trials}

\section{JCOG 8801 [11]}

Nakajima et al. [11] reported the results of an RCT comparing adjuvant chemotherapy using a combination of mitomycin $\left(\mathrm{MMC} ; 1.4 \mathrm{mg} / \mathrm{m}^{2}\right)+5-\mathrm{FU}\left(166.7 \mathrm{mg} / \mathrm{m}^{2}\right)$, 
twice weekly for 3 weeks, followed by oral administration of uracil-tegafur (UFT; $300 \mathrm{mg}$ daily) for 18 months, with surgery alone as control. They enrolled 579 patients with exclusively serosa-negative gastric cancer in the study. The 5- year survival rates of the treatment and control arms were $85.8 \%$ and $82.9 \%$, respectively. This difference was not statistically significant ( $P=0.17$; HR, 0.74; 95\% CI, 0.50-1.09). In the subgroup analysis of this study, it was suggested that this kind of adjuvant chemotherapy trial should exclude patients with T1 tumors, regardless of the pathological node positivity.

\section{JCOG 9206-1 [12]}

Nashimoto et al. [12] reported the results of an RCT comparing adjuvant chemotherapy consisting of a combination of i.v. infusion of MMC $\left(1.33 \mathrm{mg} / \mathrm{m}^{2}\right), 5-\mathrm{FU}$ $\left(166.7 \mathrm{mg} / \mathrm{m}^{2}\right)$, and cytarabine $\left(13.3 \mathrm{mg} / \mathrm{m}^{2}\right)$ twice weekly for the first 3 weeks, followed by oral UFT $\left(134 \mathrm{mg} / \mathrm{m}^{2}\right)$ for 18 months, with surgery alone as the control arm. In total, 252 patients were enrolled during 2 years. There was no significant difference between the two arms for either RFS or OS. The 5-year OS values in the test and control arms were $91.2 \%$ and $86.1 \%$, respectively. The survival curves for both RFS and OS showed a small but clear separation between the groups. Comparison of these two curves suggests that the results of this study were negative due to a too-small sample size, which had low power to detect a difference. A clinical significance of $5 \%$ superiority for this stage of gastric cancer (the patients had serosa-negative gastric cancer) should be considered in relation to the adverse events and cost of this treatment. The JCOG did not carry any out any further confirmatory study of this regimen.

\section{JCOG 9206-2 [13]}

Miyashiro et al. [13] reported the results of an RCT comparing adjuvant chemotherapy (comprising the intraperitoneal administration of cisplatin [CDDP]) and combination i.v. chemotherapy, with CDDP (70 mg/ $\left.\mathrm{m}^{2}\right)$ and 5 -FU $\left(700 \mathrm{mg} / \mathrm{m}^{2}\right)$, followed by oral administration of UFT ( $266.7 \mathrm{mg} / \mathrm{m}^{2}$ daily) for 12 months. There was no difference in OS or RFS (only $1 \%$ difference in 5-year OS). At the time when this study was planned, CDDP intraperitoneal and 5-FU + CDDP i.v. therapies were some of the most attractive ones that were thought to have the potential to improve OS and RFS. However, mainly due to the high toxicity of the intraperitoneal administration of CDDP, low compliance was a large problem in this study.

\section{National Surgical Adjuvant Study of Gastric Cancer (N-SAS-GC) [14]}

In the 1980s and 1990s, many Japanese surgeons used UFT in clinical practice without sufficient evidence that it improved OS and RFS after curative surgery. The Japanese government initiated an RCT to re-evaluate the efficacy of this drug and ordered the pharmaceutical company that produced UFT to take over this trial as a sponsored one to compare UFT $\left(360 \mathrm{mg} / \mathrm{m}^{2}, 5\right.$ days on and 2 days off) versus surgery alone for $\mathrm{pT} 2, \mathrm{pN} 1 / 2$ patients. The target population of this study was selected based on the subgroup analysis of the JCOG 8801 study. Due to very slow accrual, the sponsor and the investigators decided to stop accrual after only 190 patients had been randomized. As the original projected sample size was 500, less than half of the expected number was registered. Without any expectation, these enrolled patients were followed up, but the second interim analysis showed statistically significant differences of OS and RFS between the arms in this study. Later, with full follow up, the final survival results were reported. Fiveyear OS values after adjuvant treatment and surgery alone were $86 \%$ and $73 \%$, respectively $(P=0.017)$. The HR for OS in the chemotherapy group was $0.48(95 \%$ CI, 0.26-0.89). However, there are several criticisms of this study. The number of enrolled patients was less than half of the expected sample size. The survival rate of the control arm (surgery alone) was much lower than that in the JCOG 9201-1 study, which had been carried out in almost the same period. The 5-year RFS of the surgery-alone arm in the N-SAS-GC trial was $68 \%$, while the pT2pN1-4 subpopulation in the JCOG 9206-1 study showed a 5-year RFS of $80 \%$.

\section{Adjuvant Chemotherapy of TS-1 for Gastric Cancer (ACTS-GC) [15]}

The ACTS-GC trial was also a sponsor-led RCT, carried out to evaluate the efficacy of S-1 monotherapy as adjuvant chemotherapy after curative D2 surgery. Pathological stage II, IIIA, and IIIB patients were randomized within 6 weeks after surgery either to S-1 administration or surgery alone. The treatment regimen comprised 6week cycles, in which S-1 at $80 \mathrm{mg} / \mathrm{m}^{2}$ per day was given for 4 weeks, with no chemotherapy for the following 2 weeks. This trial showed good patient accrual of 1059 patients within 38 months. At the first planned interim analysis, the difference between the two arms was so large that the independent data and safety monitoring committee recommended to the investigators to stop the trial and open the results. The final analysis, carried out using the updated data of 6 months later, was reported in the New England Journal of Medicine. The most frequent grade $3 / 4$ toxicity was anorexia $(6 \%)$, 
followed by nausea $(3.7 \%)$ and diarrhea $(3.1 \%)$. Compliance at 6 months and at 1 year was $78 \%$ and $66 \%$, respectively. The primary endpoint, OS at 3 years, was $80.1 \%$ in the S- 1 group and $70.1 \%$ in the surgery-alone group, with an HR of 0.68 (95\% CI, 0.52-0.87). The HR of the RFS was even smaller, 0.62 (95\% CI, 0.50-0.77; $P<0.001)$. It was also reported that $\mathrm{S}-1$ significantly reduced lymph nodal $(P=0.01)$ and peritoneal $(P=$ $0.009)$ recurrence. Subgroup analysis showed a consistent HR of less than 1.0 in any subgroup, suggesting the applicability of the results to all subpopulations included in this study. This has been the first positive large highquality Japanese phase III study of adjuvant chemotherapy for curable gastric cancer to have had a strong impact on clinical practice.

\section{Comparison of Western and Japanese trials}

There have been longstanding arguments regarding the large differences in OS or RFS between Western and Japanese studies. It was often mentioned that Western and Japanese studies were treating different diseases. This is true in some aspects, but not in the majority of aspects. To date, there has been no high-quality study that has reported biological differences in gastric cancer between Western and Japanese patients. Moreover, some studies report the similarity of gastric cancers in Western and Japanese patients [16, 17]. Stage migra- tion, due to more accurate nodal staging in Japan, can explain some part of the large differences in OS and RFS[18]. To carry out fair comparison and avoid stage migration, comparison by $\mathrm{T}$ stage seems the most reliable method.

The two populations in the Intergroup 0116/SWOG 9008 study [2] and the JCOG 9206-2 trial [13] were by chance very similar in most aspects, suggesting that almost the same patient populations were treated in these two different studies. Table 2 shows the baseline characteristics of the randomized patients in these two trials. Unlike the actual features of gastric cancer patients in the United States, the majority of patients in the Intergroup 0116/SWOG 9008 study [2] had classictype antral cancer of intestinal histology. There were more patients with nodal metastasis in the United States study but there were more with diffuse cancer and more with linitis plastica in the Japanese study, resulting in a good balance in terms of prognosis. The OS in the test arm of the Intergroup 0116/SWOG 9008 study [2] was $40 \%$, while that for the entire patient cohort, including the surgery-alone group, was $61 \%$. This large difference can be explained only by a difference in treatment, i.e., D2 dissection or D1 + radiotherapy.

\section{Quality of surgery}

Subgroup analysis of the Intergroup 0116/SWOG 9008 study suggested that no benefit of the treatment was

Table 2. Comparison of Intergroup 0116/SWOG 9008, JCOG 9206-2, and MAGIC trials

\begin{tabular}{|c|c|c|c|}
\hline & Intergroup 0116 SWOG 9008 [2] & JCOG 9206-2 [13] & MAGIC [3] \\
\hline No. of patients & 281 (CRT arm) & 268 & 253 (Surgery alone) \\
\hline \multicolumn{4}{|l|}{ Tumor location } \\
\hline Antrum & $53 \%$ & $31 \%$ & NA \\
\hline Body & $24 \%$ & $32 \%$ & NA \\
\hline Cardia & $21 \%$ & $28 \%$ & $12 \%+$ Eso $14 \%$ \\
\hline Multiple & $2 \%$ & & NA \\
\hline All sites & & $9 \%$ & NA \\
\hline \multicolumn{4}{|l|}{ Histological type } \\
\hline Diffuse & 92 & 162 & NA \\
\hline Intestinal & 135 & 93 & NA \\
\hline \multicolumn{4}{|l|}{ pT stage } \\
\hline $\mathrm{T} 1$ & 14 & 5 & 16 \\
\hline $\mathrm{T} 2$ & 75 & 87 & 55 \\
\hline $\mathrm{T} 3$ & 174 & 165 & 106 \\
\hline $\mathrm{T} 4$ & 18 & 18 & 16 \\
\hline $\mathrm{T} 3+4$ & $68 \%$ & $65 \%$ & $63 \%$ \\
\hline Node-positive & $85 \%$ & $72 \%$ & $73 \%$ \\
\hline Tumor size; $\mathrm{cm}$ (median) & & 6.0 & 5.0 \\
\hline \multicolumn{4}{|l|}{ Surgery } \\
\hline D0 & $54 \%$ & $0 \%$ & $0 \%$ \\
\hline D1 & $36 \%$ & $1 \%$ & $28 \% *$ \\
\hline D2 & $19 \%$ & $99 \%$ & $53 \% *$ \\
\hline Adjuvant & $5 \mathrm{FU}+\mathrm{Leu}+\mathrm{Rad}$ & $5 \mathrm{FU}+\mathrm{CDDP}+\mathrm{UFT}$ & None-surgery alone \\
\hline 5-Year Survival & $42 \%$ & $61 \%$ & $23 \%(32 \% * *)$ \\
\hline
\end{tabular}

Eso, esophageal cancer

$* \%$ among those undergoing curative resection; $* * 5$-year survival rate among curable patients 
observed in those who underwent D2 dissection [19]. Hundahl et al. [20] made an ad-hoc analysis of the prognostic impact of hypothetical residual nodal disease, calculated using a computer program based on a large database accumulated at the National Cancer Center Hospital in Tokyo, and found that the limited dissection which had high probability of residual disease undermined the prognosis. In other words, this study clearly demonstrated that the effect of chemoradiotherapy depended on the type of surgery.

In the ACTS-GC study [15], all patients underwent D2 dissection, while only $10 \%$ and $40 \%$ of the enrolled patients underwent D2 dissection in the Intergroup 0116/SWOG 9008 study [2] and the MAGIC trial [3], respectively. It is also possible to make a comparison between the types of surgery in the MAGIC trial and the ACTS-GC study [15] or the JCOG 9206-1 study [12]. Looking at the baseline characteristics of the patients and tumors, it is hardly acceptable that the MAGIC trial has shown good results. For Japanese standards, the results of the control arm of the MAGIC trial were extremely poor, and this could have been the reason for the positive results of this study.

\section{Is radiation needed?}

The Intergroup 0116/SWOG 9008 study [2] showed a clear benefit of CRT in those who underwent less than D2 dissection. However, there remain two clinical questions. The first question is whether D1 + CRT can replace D2 + chemotherapy alone. The second one is whether CRT can improve the results of D2 dissection. The second issue is more relevant for Japanese physicians and patients, because D2 + chemotherapy is the standard of care in Japan. There is only one study, by Kim et al. [21], reporting the results of a retrospective comparison of OS between patients who underwent D2 + postoperative CRT and those with D2 surgery alone. These authors selected 446 patients as the surgery-alone group out of 3447 patients who underwent potentially curative resection. These patients fulfilled the eligibility criteria for CRT in their institution. One of the reasons for exclusion was palliative resection, which is not consistent with the description of "curative resection" for the entire group. The results of such kinds of retrospective analysis are usually far less reliable than the results of a prospective study. There were several important differences between the two groups, D2 + CRT and D2 alone, including the age of the patients, which is known to be one of the most important prognostic factors in gastric cancer patients. We should keep in mind also that this comparison was not between D2 + CRT versus D2 + chemotherapy. The OS obtained for D2 + CRT did not seem to be superior to the OS observed in the patients who underwent D2 + chemotherapy in the
ACTS-GC study [15]. In other words, the OS of those who underwent surgery alone in the study by Kim et al. [21] was far poorer than the stage-specific OS reported in the ACTS-GC study [15]. Kim and colleagues are now carrying out a single-institutional prospective RCT comparing D2 + CRT versus D2. We should wait for the results of this study. If this study shows remarkable results, Japanese physicians would have to consider the benefit of the addition of radiotherapy over D2 + chemotherapy.

\section{Future directions}

Through this review, it appears that the achievement of the ACTS-GC study [15] should be highly appreciated, and for the moment the standard of care for stage II/III gastric cancer is D2 surgery followed by postoperative adjuvant chemotherapy with S-1 for 1 year. As the results of surgery alone in Japan are acceptably good for stage II patients, the next question might be whether we can reduce the total dose and period of adjuvant treatment with S- 1 for these patients. An RCT of noninferiority design to compare 6 months' and 12 months' administration of S-1 might be an interesting trial, because the standard length of adjuvant chemotherapy for other cancers in Western countries is usually 6 months. For more advanced stages, more effective chemotherapy is expected. Careful selection of the next candidate treatment for the test arm of an adjuvant phase III trial for curable stage III gastric cancer is now ongoing; this is being done by carrying out feasibility studies using some regimens that show better OS than S-1 alone for advanced or metastatic gastric cancer. If such regimens cannot be given postoperatively, preoperative administration might be another way to go.

\section{References}

1. Oba K, Morita S, Tsuburaya A, Kodera Y, Kobayashi M, Sakamoto J. Efficacy of adjuvant chemotherapy using oral fluorinated pyrimidines for curatively resected gastric cancer: a metaanalysis of centrally randomized controlled clinical trials in Japan. J Chemother 2006;18:311-7.

2. Macdonald JS, Smalley SR, Benedetti J, Hundahl SA, Estes NC, Stemmermann GN, et al. Chemoradiotherapy after surgery compared with surgery alone for adenocarcinoma of the stomach or gastroesophageal junction. N Engl J Med 2001;345:725-30.

3. Cunningham D, Allum WH, Stenning SP, Thompson JN, van de Velde CJH, Nicolson M, et al. Perioperative chemotherapy versus surgery alone for resectable gastroesophageal cancer. N Engl J Med 2006;355:11-20.

4. Neri B, Andreoli F, Boffi B, Francesconi D, Mazzanti R, Medi F, et al. Randomized trial of adjuvant chemotherapy versus control after curative resection for gastric cancer: 5-year follow up. Br J Cancer 2001;84:878-80. 
5. Neri B, de Leonardis V, Romano S, Andreoli F, Pernice LM, Bruno L, et al. Adjuvant chemotherapy after gastric resection in node-positive cancer patients: a multicentre randomised study. $\mathrm{Br}$ J Cancer 1996;73:549-52.

6. Bajetta E, Buzzoni R, Mariani L, Beretta E, Bozzetti F, Bordogna $\mathrm{G}$, et al. Adjuvant chemotherapy in gastric cancer: 5-year results of a randomized study by the Italian Trials in Medical Oncology (ITMO) Group. Ann Oncol 2002;13:299-307.

7. Chipponi J, Hugier M, Pezet D, Basso N, Hay JM, Quandalle P, et al. Randomized trial of adjuvant chemotherapy after curative resection for gastric cancer. Am J Surg 2004;187:440-5.

8. Bouché O, Ychou M, Burtin P, Bedenne L, Ducreux M, Lebreton $\mathrm{M}$, et al. Adjuvant chemotherapy with 5-fluorouracil and cisplatin compared with surgery alone for gastric cancer; 7-year results of the FFCD randomized phase III trial (8801). Ann Oncol 2005; 16:1488-97.

9. Nitti D, Wils J, Dos Santos JG, Fountzilas G, Conte PF, Sava C, et al. Randomized phase III trials of adjuvant FAMTX or FEMTX compared with surgery alone in resected gastric cancer. A combined analysis of the EORTC GI Group and the ICCG. Ann Oncol 2006;17:262-9.

10. De Vita F, Giuliani F, Orditura M, Galizia G, Di Martino N, Montemurro F, et al. Adjuvant chemotherapy with epirubicin, leucovorin, 5-fluorouracil and etoposide regimen in resected gastric cancer patients: a randomized phase IIItrial by the Gruppo Oncologico Italia Meridionale (GOIM 9602 Study). Ann Oncol 2007:18:1354-8.

11. Nakajima T, Nashimoto A, Kitamura M, Kito T, Iwanaga T, Okabayashi K, et al. Adjuvant mitomycin and fluorouracil followed by oral uracil plus tegafur in serosa-negative gastric cancer: a randomized trial. Lancet 1999;354:273-7.

12. Nashimoto A, Nakajima T, Furukawa H, Kitamura M, Kinoshita T, Yamamura Y, et al. Randomized trial of adjuvant chemotherapy with mitomycin, fluorouracil, and cytosine arabinoside followed by oral fluorouracil in serosa-negative gastric cancer; Japan Clinical Oncology Group 9206-1. J Clin Oncol 2003;21:2282-7.

13. Miyashiro I, Furukawa H, Sasako M, Yamamoto S, Nashimoto A, Nakajima T, et al. No survival benefit with adjuvant chemotherapy for serosa-positive gastric cancer: randomized trial of adjuvant chemotherapy with cisplatin followed by oral fluorouracil (UFT) in serosa-positive gastric cancer. Presented at the 2005 Gastrointestinal Cancers Symposium; 2005 January 27-29; Hollywood, FL, USA. Alexandria, VA, American Society of Clinical Oncology; 2005.

14. Nakajima T, Kinoshita T, Nashimoto A, Sairenji M, Yamaguchi T, Sakamoto J, et al. Randomized controlled study of adjuvant uracil-tegafur versus surgery alone for serosa-negative, locally advanced gastric cancer. Br J Surg 2007;94:1468-76.

15. Sakuramoto S, Sasako M, Yamaguchi T, Kinoshita T, Fujii M, Nashimoto A, et al. Adjuvant chemotherapy for gastric cancer with S-1, an oral fluoropyrimidine. N Engl J Med 2007;357: 1810-20.

16. McCulloch PG, Ochiai A, O’Dowd GM, Nash JR, Sasako M, Hirohashi S. Comparison of the molecular genetics of c-erb-B2 and p53 expression in stomach cancer in Britain and Japan. Cancer 1995;75:920-5.

17. Bonenkamp JJ, van de Velde CJ, Kampschöer GH, Hermans J, Hermanek P, Bemelmans M, et al. Comparison of factors influencing the prognosis of Japanese, German, and Dutch gastric cancer patients. World J Surg 1993;17:410-5.

18. Bunt AMG, Hermans J, Smit VT, van de Velde CJ, Fleuren GJ, Bruijn JA. Surgical/pathological stage migration confounds comparisons of gastric cancer survival rates between Japan and western countries. J Clin Oncol 1995;13:19-25.

19. Macdonald JS, Smalley S, Benedetti J, Estes N, Haller D, Ajani JA, et al. Presented at the 2004 Gastrointestinal Cancers Symposium; 2004 Jan 22-24; San Francisco, CA, USA. Alexandria, VA, American Society of Clinical Oncology; 2004.

20. Hundahl SA, Macdonald JS, Benedetti JB, Fitzsimmons T. Surgical treatment variation in a prospective randomized trial of chemoradiotherapy in gastric cancer: the effect of undertreatment. Ann Surg Oncol 2002;9:278-86.

21. Kim SK, Lim DH, Lee JY, Kang WK, Macdonald JS, Park CH, et al. An observational study suggesting clinical benefit for adjuvant postoperative chemoradiotherapy in a population of over 500 cases after gastric resection with D2 nodal dissection for adenocarcinoma of the stomach. Int J Radiat Oncol Biol Phys 2005;63:1279-85. 